Revista de Psicología Vol. 33 (2), 2015 (ISSN 0254-9247)

\title{
Apoyo familiar en escolares de alta capacidad intelectual de diferentes contextos socioeducativos ${ }^{1}$
}

\author{
Doris Castellanos Simons², Aldo Bazán Ramírez \\ Aldo Mauricio Ferrari Belmont ${ }^{4}$ y César Alberto Hernández Rodríguez ${ }^{5}$ \\ Universidad Autónoma del Estado de México
}

El objetivo del trabajo fue analizar la percepción del apoyo familiar al aprendizaje en 79 alumnos de 6to grado con aptitud intelectual superior de tres contextos educativos (escuela urbana pública y privada, y rural indígena) en Morelos, México. Los alumnos respondieron la prueba SAGES-2 (Johnson \& Corn, 2001) y el Cuestionario de Apoyo Familiar de Bazán \& Domínguez (2009). Solamente se encontraron diferencias significativas entre los contextos analizados en la percepción de la comunicación regular con maestros relacionada con la materia Espańol, que fue percibida con mayor frecuencia en el contexto de la escuela privada, respecto al público indígena, independientemente del área de capacidades detectada. Se enfatiza la urgencia de investigar las variables del contexto socioeconómico y familiar para la comprensión de la manifestación, desarrollo y educación de las altas capacidades en alumnos de entornos culturales diferentes.

Palabras clave: altas capacidades, apoyo familiar, contexto socioeducativo.

\section{Family support in high ability students from different socio-educational contexts}

This work aimed to analyze the perceptions of family support of school learning in 79 high intellectual ability $6^{\text {th }}$ grade students from three educational contexts in Morelos, Mexico: urban (public, private) and rural indigenous schools. The students answered the SAGES-2 (Johnson \& Corn, 2001) and the Family Support Questionnaire of Bazán \& Domínguez (2009). Significant differences were only found between the three contexts in

1 Este trabajo presenta resultados parciales de una investigación financiada por el Consejo Nacional de Ciencia y Tecnología (CONACYT) de México (Proyecto No. 151981) dirigida por el doctor Aldo Bazán Ramírez.

2 Doctora en Psicología (RUN), es profesora investigadora en el Centro de Investigación Transdisciplinar en Psicología de la UAEM. Dirección postal: Pico de Orizaba No. 1. Col. Los Volcanes, Cuernavaca, Mor. C.P. 62350 México. Contacto: dcastellanos@uaem.mx

3 Doctor en Psicología (UNAM), es profesor investigador en el Centro de Investigación Transdisciplinar en Psicología de la UAEM. Contacto: abazanramirez@gmail.com

4 Licenciado en Psicología (UAEM). Contacto: aferrab@gmail.com

5 Licenciado en Psicología (UAEM). Contacto: cesalber.herod@gmail.com 
the perception of regular communication with teachers in Language (Spanish), which was perceived more frequently in students from private schools, regardless of the area of abilities. This communication was perceived the least in students from rural indigenous schools. The results emphasize the importance of family and socioeconomic variables to understand the manifestation, development, and education of high ability students from different cultural contexts.

Keywords: High abilities, family support, socio-educational context. 
Los modelos actuales sobre el desarrollo de las altas capacidades y talentos insisten en el papel decisivo de los factores sociales y contextuales en al desarrollo de las potencialidades de niños, adolescentes y jóvenes, y en general en la determinación sociohistórica y cultural de las mismas. Más allá de la diversidad de enfoques y definiciones sobre estas categorías, y de la discusión en torno a los fenómenos designados por ellas, la tendencia general en el desarrollo de la investigación en el campo ha sido la búsqueda de abordajes sistémicos, que den cuenta de la naturaleza multifactorial y compleja de estos fenómenos, que respondan a la "dominancia cultural", y que se inscriban en un paradigma caracterizado por la sensibilidad hacia la diversidad cultural en la investigación relativa a la "ciencia del talento".

Los más difundidos enfoques sobre el desarrollo de la alta capacidad (Gagné, 2010), y en México, el modelo asumido por la Secretaría de Educación Pública (SEP, 2006), conciben a la familia como una pieza clave, una de las influencias educativas más poderosas para la transformación del potencial en talentos actuantes, no solo por constituir el primer contexto de socialización del ser humano, sino porque aporta "ingredientes" básicos a la educación de los niños con altas capacidades, como conocimientos, ambiente de libertad, seguridad, atención personalizada, educación permanente (De la Torre \& Pérez, 2006); para Freeman (2010), constituye el contexto esencial para las capacidades y talentos en toda la riqueza y diversidad de influencias y oportunidades que representa.

\section{Contexto familia y desarrollo de las altas capacidades}

Existe una amplia investigación a nivel internacional que reporta que el peso de algunos de los factores socioculturales es crítico en el proceso de transformación del potencial de los sujetos a capacidades 
y talentos actuales. Tal es el caso de la investigación centrada en el impacto de las variables del contexto familiar, entre ellas, las creencias y expectativas de los padres respecto a las potencialidades de los hijos y al rol de la escuela en su estimulación, los patrones o pautas de interacción y comunicación en el seno de la familia, y en particular, el apoyo familiar (Colangelo \& Assoluline, 2000; Gagné, 2010; Jiménez, Murga, Gil, Téllez \& Trillo; 2010; López-Aymes, Acuña \& Durán, 2013; Martínez \& Girardo, 2012; Ordaz \& Acle, 2012; Pérez, 2004; Soriano, 2013). De hecho, la alta capacidad es vista desde esta perspectiva como un constructo que integra dimensiones personales (no solo cognitivas), socioculturales y contextuales en una compleja interacción (Jiménez et al., 2010; Plucker \& Barab, 2005), y que en su tránsito hacia su manifestación en talentos en áreas particulares, se expresa como una configuración dinámica y "un resultado de la integración (estructural y funcional) de un conjunto heterogéneo de procesos, cualidades y formaciones psicológicas" (Castellanos, 2003, p. 3) en su interacción con un contexto sociohistóricamente condicionado.

Jiménez y otros (2010) han insistido en sus estudios en las características sociológicas de los estudiantes con capacidad sobresaliente, y en particular, en el análisis de sus familias; estos autores concluyen que los factores determinantes en la alta capacidad y el alto rendimiento parecen ser la educación familiar recibida, además del esfuerzo y la determinación del propio alumno. Los investigadores encontraron que los estudiantes universitarios identificados como sobresalientes en sus programas, proceden en gran parte de la clase media y media alta, de familias estables y maduras que invierten de manera constante medios y tiempo en la formación de los hijos, para potenciar en ellos recursos personales en determinados campos desarrollados paralelamente, de manera extracurricular a su propia formación académica (idiomas, música, danza, informática, etc.). Los estudiantes de clases sociales bajas tenían prácticamente vetado el camino hacia los llamados Premios Extraordinarios de Bachillerato, lo que determinaba evidentemente la existencia de un obstáculo a una de las 
principales vías que facilitan la propia identificación, la manifestación y el desarrollo de su potencial intelectual. No obstante estos patrones, encontraron que lo que más distinguía a estas familias eran sus expectativas, los roles promovidos o cuestionados, y los tratamientos proporcionados a sus hijos.

Por su parte, Castellanos y colaboradores (2004), en investigaciones con estudiantes cubanos académicamente talentosos en educación básica, media superior y universitaria, encontraron contextos familiares orientados a la estimulación del potencial de sus hijos, independientemente de que, en ocasiones, resultase obvia la escasez de recursos económicos y materiales con que contaban. En este sentido, los autores caracterizaron los entornos familiares "desarrolladores": familias con creencias y expectativas relativamente altas y positivas respecto a las potencialidades de sus hijos, con un nivel de información y/o sensibilización al menos básico sobre la temática, la alta capacidad y su educación, que hacen patente su apoyo a los sujetos desde lo afectivo, lo material o lo instrumental (a veces los tres), creando estrategias y oportunidades de diversa naturaleza para su potenciación.

Otros estudios actuales han demostrado que no necesariamente los alumnos sobresalientes y con talentos provienen de contextos familiares con un óptimo perfil socioeconómico y cultural (Borges, Hernández \& Rodríguez, 2006; López-Aymes, Acuña \& Mercado, 2013; Ordaz \& Acle, 2012), y más bien destacan la heterogeneidad de las familias y la existencia de evidencias a veces contradictorias respecto a su influencia. En este sentido, si bien a veces se reporta la manifestación de actitudes positivas y disposición especial de las mismas a brindar a sus hijos reconocimiento y apoyo, en otros trabajos, como los de Martínez y Guirardo (2012) se ha documentado la existencia de familias que manifiestan grandes conflictos al respecto: declaran no poder prestar ayuda a sus hijos sobresalientes, y no dedicarles tiempo, entre otras cosas debido al hecho de sentirse incapaces o no suficientemente preparados de cara al reconocimiento de estas altas capacidades. 
En particular, en el contexto mexicano, Valdés, Vera y Yañez (2012) han sistematizado también un amplio rango de reacciones diversas de las familias ante la conciencia del alto potencial y/o los talentos en los hijos: estas van desde el claro entusiasmo, orgullo y satisfacción, asunción de responsabilidad, y esfuerzos por instrumentar actividades de enriquecimiento para ellos, pasando por efectos como: sentimientos de desorientación, inseguridad, miedos por no saber cómo responder a sus demandas, preocupaciones por su ajuste personal, social, por su proyecto de vida futura, hasta llegar, finalmente, a la propia negación del talento y la potencialidad de los niños, adolescentes y jóvenes. Los resultados de sus investigaciones son congruentes con lo encontrado por otros autores en familias mexicanas que tienen hijos con altas capacidades intelectuales, aptitudes sobresalientes y talentos en áreas particulares (Soriano \& Castellanos, 2014; López-Aymes et al., 2013; Gómez \& Valadez, 2010; Soriano, 2013).

Por otra parte, los estudios de Arencibia (2010) con alumnos con talentos académicos en contextos de pobreza, en estratos socioeconómicos bajos y medios bajo, también demuestran la importancia de la identificación temprana del potencial para invertir esfuerzos educativos, y de mostrar una sensibilidad especial ante la problemática de este tipo de alumnado que se caracteriza por la combinación de factores como: pobres recursos educacionales, estrategias parentales deficientes, problemas de salud, ambientes familiares desorganizados, entre otros.

Puede decirse que en general, aunque se ha enfatizado en la necesidad de desarrollar estudios que aborden el papel de los factores psicosociales y contextuales en el desarrollo de los individuos con altas potencialidades, muchas de las investigaciones que se realizan en la actualidad se ubican más en una perspectiva individual, que resalta el funcionamiento personal de estos sujetos, con insuficiente profundización en las influencias socioeducativas, $y$ en las condiciones socioculturales sobre la base de las cuales los talentos se construyen y se desarrollan (Gagné, 2010; Renzulli \& Reiss, 2000; Sternberg, 2001; Plucker \& Barab, 2005; Ziegler \& Heller, 2000), de aquí la importancia de los aportes de aquellos estudios que abordan el impacto de la familia como contexto complejo de desarrollo. 
Apoyo familiar en escolares de alta capacidad intelectual de diferentes contextos... / Castellanos et al.

\section{Apoyo familiar al aprendizaje escolar}

Desde una perspectiva un tanto similar, la problemática del logro académico o del desempeño escolar del alumnado se ha sido estudiada ampliamente en su relación con diferentes factores que los facilitan o dificultan (Martínez, 2004), en particular, los factores de contexto (Cervini, 2003, 2003; OCDE, 2003; Purkey \& Smith, 1983; Reimers \& Cárdenas, 2007; Schmelkes, 2003).

Respecto al contexto sociocultural y económico, la educación pública mexicana enfrenta problemas sustanciales que tienen que ver todavía con problemas de acceso y oportunidades equitativas a la educación, y por otro lado, con los problemas de la baja calidad educativa. Los datos sobre resultados educativos en la Educación Básica en México muestran marcadas diferencias por estrato socioeconómico, tanto en los niveles de ejecución como en los puntajes obtenidos: ambos son más altos para alumnos de escuelas privadas, seguidos por alumnos de escuelas públicas urbanas, mientras que los peores logros y niveles de dominio se encuentran en los alumnos de programas compensatorios como el Consejo Nacional de Fomento Educativo (CONAFE), los del sistema de educación indígena, o los de escuelas públicas rurales. Así por ejemplo, Backhoff, Sánchez, Peón y Andrade (2010) y Backhoff, (2011), utilizando evaluaciones realizadas por la Secretaría de Educación Pública (SEP) en el año 2000 y replicándolas en el año 2005, encontraron que los resultados en las mismas fueron explicados por las diferencias que existen entre las oportunidades de aprendizaje y el capital cultural de las familias de los distintos estratos y modalidades escolares en México.

Aunque existe una gran diversidad en las familias, existe ya todo un cuerpo de conocimientos acerca las variables implicadas en el ejercicio de la participación, y de relaciones de colaboración entre familia y escuela (Huerta, 2009). Resulta importante conocer cuál es el papel que desempeñan los padres en el apoyo a los estudiantes y a su desempeño escolar, identificando con mayor precisión la naturaleza de este apoyo, o lo que en esta investigación se designa como estrategias de apoyo, 
con un énfasis en la función de "padres como maestros" (Martiniello, 1999, citado en Alacalay, Milicic \& Torretti, 2005), y en dimensiones abordadas por autores como Epstein (2001, citado en Rivas, 2007) y Grolnick y Slowiaczek (1994) como son: la participación cognitivo intelectual, la participación personal, el monitoreo (monitoring) y la implicación (involvement).

Bazán, Sánchez y Castañeda (2007) han enfatizado en particular, en el involucramiento de los padres en actividades relacionadas con el desarrollo académico de los hijos, aquellas que los miembros de la familia realizan para apoyar los esfuerzos de la escuela en el aprendizaje escolar, de manera complementaria a las propias prácticas didácticas, de enseñanza-aprendizaje que establece el centro, designándolas con el término "apoyo familiar". Los autores señalan que el involucramiento de las familias en estas prácticas depende de diversos factores, entre ellos, de sus expectativas y creencias acerca de cuánto puedan resultar los hijos beneficiados con aquellas, y en qué medida pueden hacer una "diferencia significativa en la escuela a favor de sus hijos" (p. 704), así como su eficacia (o capacidad) para desarrollarlas. En este sentido, esas creencias y juicios comprenden igualmente la autovaloración de la propia competencia para apoyar en las tareas escolares y el desarrollo académico de los hijos.

Reconociendo los múltiples abordajes teóricos que ha tenido el concepto de apoyo familiar, en la presente investigación se definirá de manera puntual como el grado de ayuda, asesoría, y supervisión, que los padres brindan a sus hijos a través de una serie de actividades, con el fin de mejorar su dominio en las habilidades a desarrollar y en las tareas a realizar en la escuela (Bazán et al., 2007). El apoyo familiar se expresa mediante diversas conductas, actividades y estrategias que las familias despliegan dentro y fuera de las escuelas: desde la ejecución de las obligaciones educativas con los hijos y el cumplimiento de determinadas funciones establecidas dentro de la escuela, hasta el apoyo directo a sus hijos al otorgar espacio y tiempo para la ejecución de las tareas, proveer de herramientas de trabajo, brindar acompańamiento, supervisión y seguimiento en los deberes escolares, entre otras. 
Apoyo familiar en escolares de alta capacidad intelectual de diferentes contextos... / Castellanos et al.

Se infiere también de esto que el apoyo familiar posee igualmente una dimensión de subjetividad, que comprende las expectativas y la percepción que tienen los padres sobre sus propias conductas respecto a los hijos, así como también la perspectiva de los hijos sobre estas conductas de los padres, sobre su intencionalidad y pertinencia, y la forma en que comprenden y dan significado a las interacciones que se establecen entre ellos, en este y otros campos de su actividad conjunta.

Un cuerpo relativamente amplio de trabajos han analizado las percepciones, expectativas, creencias y estereotipos de los padres respecto a las características y necesidades de sus hijos con aptitudes sobresalientes, y en particular, sus autovaloraciones sobre el grado y la manera en que apoyan a sus hijos, estimulando el desarrollo de sus potencialidades por medio de diversas estrategias; sin embargo, otras investigaciones se han centrado en las opiniones de los alumnos (Jiménez et al., 2010; Martínez, 2004; Martínez \& Guirardo, 2012; Pérez, 2004).

Entre los estudios desarrollados en esta última dirección destacan los de Borges, Hernández y Rodríguez (2006), y de Hernández y Borges (2010), quienes encontraron una gran diversidad en el contexto familiar de más de 1000 alumnos de Educación Secundaria Obligatoria (ESO) con altas capacidades intelectuales que participaron en sus estudios, en colegios públicos, privados y concertados de Canarias. Resulta singular que en la muestra se evidenció que estos provienen de familias con alto poder adquisitivo y nivel socioeconómico superior, encontrándose una mayor proporción de estudiantes identificados por su alto potencial intelectual en escuelas privadas que en escuelas públicas. En sus estudios, no se encontraron diferencias significativas entre las valoraciones que hacen los alumnos con altas capacidades y las de sus compañeros de capacidad promedio sobre diversos aspectos de la interacción y el apoyo familiar, como: apoyo y supervisión de tareas escolares, poner a su disposición medios de apoyo al estudio (desde garantizar un lugar apropiado hasta proveer recursos materiales); tampoco difirieron sus valoraciones acerca de aspectos del entorno escolar. En general, los estudiantes sobresalientes percibieron a sus progenitores como preocupados por sus actividades y logros académicos, aunque 
concordaron en que les permiten una cierta autonomía respecto a la realización de sus tareas. Esto permitió destacar un hecho que se relaciona con lo observado en estudios anteriores: la presencia de actitudes educadoras que fomentan la autonomía y la independencia (Borges, Hernández-Jorge \& Rodríguez-Naveiras, 2006) en muchas de estas familias. Por otra parte, una proporción significativa de los alumnos manifestaron no haber observado una estrecha relación entre padres y profesores, y sostuvieron en este sentido una visión muy crítica y realista de su entorno, y en concreto de sus familias.

En general, puede afirmarse que existe aún poca investigación sobre la percepción que tienen los alumnos con alta capacidad intelectual sobre el apoyo que reciben de la familia en el desarrollo de sus actividades escolares. Mucho más interés han tenido hasta el momento los trabajos dirigidos a determinar las características sociodemográficas y las condiciones socioculturales de estas familias. Resulta pertinente, entonces, conocer las opiniones que tiene este tipo de alumnado sobre el apoyo que reciben de sus padres para acompańar su proceso educativo y su aprendizaje escolar en el contexto no escolarizado, así como analizar las posibles diferencias que se generan en diferentes contextos socioeconómicos y culturales.

El objetivo general del estudio fue precisamente comparar la percepción del apoyo familiar al aprendizaje escolar en escolares de 6to grado con aptitud intelectual superior provenientes de escuelas ubicadas en tres contextos socioeducativos diferentes: privado, público (urbano), y rural (indígena).

En el presente trabajo se utiliza el término "alta capacidad" como sinónimo de aptitud sobresaliente, reservado para indicar la integración de un conjunto específico de habilidades y competencias generales (ejemplo, capacidades de razonamiento, solución de problemas, etc.) o específicas (de diferentes áreas, dominios y contenidos), que destacan en el contexto de un grupo etáreo y educativo. Se analiza la alta capacidad intelectual en el ámbito académico, a partir de su expresión en la ejecución de alumnos del primer grado de secundaria en la resolución de tareas dirigidas a identificar sus capacidades y competencias 
en dos áreas curriculares (Matemáticas/Ciencias Naturales, y Lengua/ Literatura/Humanidades), y en el área de Razonamiento.

El apoyo familiar se estudia como el involucramiento de los padres en el aprendizaje de sus hijos (Bazán et al., 2007), mediante acciones concretas tendientes a prestar asistencia en la realización de las tareas escolares, repasar y evaluar, facilitar tiempo y espacio para su realización, y establecer comunicación con los maestros y la escuela. Entre las características más importantes de las familias de niños con altas capacidades intelectuales se han destacado, además de la estabilidad familiar y los recursos materiales o educativos que posean, el apoyo que se presta a los miembros (Olszewski-Kubilius, 2008, citada por Hernández \& Borges, 2010).

Adicionalmente, se explora la influencia del contexto socioeducativo en esta relación, analizando el comportamiento de estas variables en tres contextos representados por escuelas del área urbana-pública (EP) y privada (EPV) - y rural indígena (EI). En este caso, las condiciones socioeconómicas y geográficas del área o comunidad, así como el nivel socioeconómico de la población asistente a la escuela en cuestión, han sido considerados como indicadores de las modalidades educativas que configuran diferentes contextos socioeducativos con diferente capital cultural escolar (Backhoff, 2011; Bazán et al., 2007).

\section{Método}

La presente investigación es un estudio no experimental, transversal, de tipo descriptivo, con un enfoque cuantitativo.

\section{Participantes}

La población del estudio estuvo conformada por 429 alumnos de 6 to grado ( $49.6 \%$ de ellos, mujeres y $50.4 \%$ hombres) con edades entre 11 y 13 ańos, provenientes de tres tipos de escuelas: 2 urbanas privadas $(\mathrm{n}=84), 3$ urbanas públicas $(\mathrm{n}=158)$ de la zona capitalina de Cuernavaca, Morelos, y 3 escuelas rurales indígenas $(n=187)$ 
ubicada en un municipio aledaño a la capital del Estado, incorporadas al proyecto financiado por CONACYT "Influencia del apoyo familiar y variables asociadas sobre el logro académico en tres contextos educativos en Morelos y Sonora".

Por medio de un procedimiento de criba, a partir de los resultados obtenidos en la prueba para la evaluación de aptitudes sobresalientes SAGES-2, se seleccionaron aquellos alumnos que obtuvieron un cociente igual o superior a 120 ("probable o muy probable la presencia de aptitud intelectual sobresaliente") en al menos una de las tres escalas del instrumento. De la población general, resultó seleccionado a partir de este criterio solo el $18.4 \%$ de los alumnos $(\mathrm{n}=79)$; de ellos, un $8.15 \%$ proveniente de las escuelas privadas, $7.22 \%$ de las escuelas públicas, y $3.03 \%$ de las escuelas rurales.

A partir de este procedimiento, la muestra quedó conformada por 79 estudiantes ( 47 hombres y 32 mujeres) de 6to grado con edad promedio de 11.7 , de los cuales 35 pertenecen al contexto urbano privado (44.3\%), 31 al contexto urbano público (39.2\%), y 13 (16.5\%) al rural indígena, lo que representó un 34\% del total de la población estudiada. Del total de los 79 alumnos, el $67.1 \%$ resultó con aptitud intelectual sobresaliente en 1 de las 3 áreas que evalúa la prueba, mientras que el 22.8 mostró AS en 2 de las áreas, y solo un 10.1\% resultó sobresaliente en las 3 áreas o dimensiones evaluadas (ver Tabla 1).

La escuela privada urbana recibe alumnos de familias con nivel socioeconómico medio superior y alto, y está ubicada en una zona residencial de la capital. Las familias de los estudiantes de la escuela pública son de nivel socioeconómico medio y bajo. En ambas, el nivel cultural o educativo de los padres de familias presenta alta variabilidad. La escuela rural indígena se ubica en una comunidad de un municipio situado a $10 \mathrm{~km}$ de la capital del Estado de Morelos; las familias en su mayoría cuentan con bajos o muy bajos ingresos, son agricultores o artesanos, una pequeña parte de ellos solo dominan náhuatl u otra lengua indígena, aunque también los padres presentan diferentes niveles de escolaridad. La propia escuela es bilingüe, en las clases se trabaja en idioma español y náhuatl. 
Apoyo familiar en escolares de alta capacidad intelectual de diferentes contextos... / Castellanos et al.

\section{Tabla 1}

Distribución de los grupos de alta capacidad (Perfil Altas Capacidades) por contexto (frecuencias y porcentajes)

\begin{tabular}{|c|c|c|c|c|c|c|}
\hline & & & \multicolumn{3}{|c|}{ Perfil Alta Capacidad (AC) } & \multirow[t]{2}{*}{ Total } \\
\hline & & & $\begin{array}{c}\mathrm{AC} \text { en } \\
1 \text { área }\end{array}$ & $\begin{array}{l}\mathrm{AC} \text { en } \\
2 \text { áreas }\end{array}$ & $\begin{array}{l}\mathrm{AC} \text { en } \\
3 \text { áreas }\end{array}$ & \\
\hline \multirow{9}{*}{ Contexto } & \multirow{3}{*}{$\begin{array}{l}\text { Urbano } \\
\text { Privado }\end{array}$} & Frecuencia & 23 & 6 & 6 & 35 \\
\hline & & $\%$ Perfil AC & $43.4 \%$ & $33.3 \%$ & $75.0 \%$ & $44.3 \%$ \\
\hline & & $\%$ del Total & $29.1 \%$ & $7.6 \%$ & $7.6 \%$ & $44.3 \%$ \\
\hline & \multirow{3}{*}{$\begin{array}{l}\text { Urbano } \\
\text { Público }\end{array}$} & Frecuencia & 21 & 8 & 2 & 31 \\
\hline & & $\%$ Perfil AC & $39.6 \%$ & $44.4 \%$ & $25.0 \%$ & $39.2 \%$ \\
\hline & & $\%$ total & $26.6 \%$ & $10.1 \%$ & $2.5 \%$ & $39.2 \%$ \\
\hline & \multirow{3}{*}{$\begin{array}{l}\text { Rural } \\
\text { indígena }\end{array}$} & Frecuencia & 9 & 4 & 0 & 13 \\
\hline & & $\%$ Perfil AC & $17.0 \%$ & $22.2 \%$ & $0.0 \%$ & $16.5 \%$ \\
\hline & & $\%$ total & $11.4 \%$ & $5.1 \%$ & $0.0 \%$ & $16.5 \%$ \\
\hline \multirow{2}{*}{$\begin{array}{l}\text { Total } \\
\% \text { del total }\end{array}$} & & Frecuencia & 53 & 18 & 8 & 79 \\
\hline & & $67.1 \%$ & $22.8 \%$ & $10.1 \%$ & $100.0 \%$ & \\
\hline
\end{tabular}

\section{Medidas e instrumentos de medición}

a) SAGES-2. Evaluación inicial para estudiantes con aptitudes sobresalientes. Nivel 2 (4to hasta Secundaria). Se utilizó la prueba SAGES, en inglés Screening Assessment for Gifted Elementary and Middle School Students (2da Edición), de Johnsen, y Corn (2001, citado en Orta \& Sánchez, 2003), validada y estandarizada en México por Orta y Sánchez (2003). Se trata de una prueba diseñada para evaluar la capacidad de razonamiento así como los conocimientos generales de niños y adolescentes con aptitudes sobresalientes, e identificar este tipo de población en el contexto escolar. Entre los principales usos de la SAGES- 2, según Valdés y colaboradores (2012), esta sirve como un instrumento de medición en estudios que investigan la capacidad académica e intelectual. Las normas del Sages -2 en México (Orta \& Sánchez, 2003) derivaron de una muestra de 402 estudiantes de dos 
regiones del país: zonas metropolitanas —urbanas y suburbanas— de la Ciudad de México y estudiantes de Mérida, Yucatán (asumida como representativa de la provincia mexicana), por el método de los conglomerados, en alumnos de escuelas públicas y privadas. Se aplicó el segundo nivel, recomendado para alumnos desde cuarto grado de primaria hasta secundaria (SAGES 2).

La prueba consta de tres subescalas: 1) Matemáticas / Ciencias Naturales: sus reactivos se vinculan con dos áreas académicas de naturaleza lógica y técnica. Lengua y literatura / Ciencias sociales, que incluye tareas de naturaleza lingüistica, las áreas evaluadas en esta subescala han sido obtenidas de diversos libros de texto y literatura; al igual que en el caso de la subescala 1; 3) Razonamiento: evalúa la capacidad para percibir relaciones y resolver tareas nuevas a través de tareas en forma de analogía que buscan que el sujeto reconozca patrones y aplique habilidades como: clasificación, discriminación, deducción y reconocimiento detallado. Las dos primeras escalas comprenden 30 reactivos cada una, con formato de opción múltiple (5 posibles respuestas, 1 correcta), mientras que la última tiene 35 reactivos. La prueba proporciona puntuaciones directas, cocientes y percentiles. En el presente estudio, se obtuvo un coeficiente de confiabilidad Alpha de Cronbach aceptable para la escala total (.89) así como para las subescalas $(.76, .68 ; .85$ para las subescalas 1,2 y 3 respectivamente).

b) Percepción del apoyo familiar: el cuestionario de evaluación del apoyo familiar (Bazán et al., 2007; Domínguez, 2008; Domínguez, 2011) tiene como finalidad obtener información relacionada con el apoyo que los padres brindan a sus hijos en las materias de Español y Matemáticas, dos asignaturas claves en el currículo escolar que impactan de manera esencial las evaluaciones del logro académico en la población escolar, en particular, en la educación básica. Existen dos versiones del cuestionario: la versión para padres y la versión para el alumno, que utilizan los mismos indicadores y las mismas preguntas, variando solamente la forma de plantear las preguntas. En el presente estudio se reportó solamente el resultado de la aplicación de la versión para alumnos. El cuestionario consta de 19 reactivos, distribuidos en cuatro 
dimensiones: 1) Asistencia o apoyo en tareas escolares, 2) Tiempo y espacio proporcionado para el estudio, 3) Comunicación regular con los docentes y directivos, y 4) Repaso y evaluación; se contestan para la materia de Espańol y para la de Matemática separadamente, en una escala Likert con 5 opciones que miden la frecuencia de las conductas analizadas: a) nunca, b) casi nunca, c) algunas veces, d) casi siempre e) siempre, con excepción de uno de los ítems (tiempo dedicado por los padres al apoyo en las tareas escolares). Los resultados se ofrecen en un rango de valores entre 0 y 4 que pueden interpretarse, según los autores, como un indicador de la percepción sobre la frecuencia con que los padres desarrollan cierto tipo de comportamientos de apoyo a sus hijos; los valores mayores indican una frecuencia mayor. Igualmente, se calcula un promedio general para cada una de las 4 dimensiones del apoyo. El cuestionario (Domínguez, 2009) ha mostrado validez convergente y de constructo, en su aplicación a tres fuentes de información (padres, madres, y alumnos), e índices de consistencia interna aceptables en sus cuatro categorías, que van de (coeficientes de .78, .51, .65, y .73 respectivamente). En la presente investigación se obtuvieron coeficientes de confiabilidad en español de $.77, .55, .72$ y .61, y en Matemática de $.58, .62, .72$ y .77 , con totales de .87 en ambas categorías.

\section{Procedimiento}

Después de establecer el contacto con las escuelas y de obtener la autorización de estas, se convocó una junta propiciada por la dirección de los centros con los padres de los alumnos seleccionados en la que firmaron un consentimiento informado para autorizar la participación de sus hijos en el proyecto. Se administraron a todo el alumnado de 6to año las tres subescalas del SAGES-2 en dos sesiones diferentes realizadas en las aulas. En la primera sesión los alumnos completaron la Subescala 1 (Matemáticas y Ciencias Naturales) y la Subescala 3: Razonamiento, con un tiempo máximo de 40 minutos en cada escala. En la segunda sesión, completaron la Subescala 2 (Lengua, Literatura y Humanidades) en 40 minutos como máximo, e igualmente, el Cuestionario de Apoyo Familiar. Se trabajó paralelamente en las tres escuelas 
con el apoyo de un equipo de investigación conformado por tesistas de la Licenciatura en Psicología y de la Maestría en Psicología de la UAEM, que habían recibido capacitación previa.

\section{Análisis y procesamiento de la información}

Los datos fueron analizados con ayuda del paquete estadístico SPSS versión 20. Se utilizaron estadísticas descriptivas para examinar el comportamiento de las variables analizadas en la muestra general y en los diferentes subgrupos. Para el cálculo de las puntuaciones cocientes y percentiles en la prueba SAGES-2 se utilizaron los baremos aportados en el manual de Johnsen y Corn (2003) para la población escolar general mexicana. Las posibles diferencias por sexo en las áreas de capacidad intelectual evaluadas en el SAGES 2 y en percepción de apoyo familiar en las categorías finales se examinaron por medio de las pruebas de comparación de medias para muestras independientes. Igualmente se aplicó un análisis de varianza simple para examinar las diferencias entre los alumnos provenientes de los tres contextos en las ocho categorías de apoyo familia, y posteriormente se realizaron pruebas post hoc de Comparaciones Múltiples de Bonferroni para identificar dónde se ubicaban las diferencias. Finalmente, se llevó a cabo el cálculo de correlaciones para examinar las asociaciones entre las diferentes variables, en la muestra general y dentro cada uno de los contextos educativos. En todas las pruebas se utilizó un nivel de significación de .05.

\section{Resultados}

A continuación se resumen los principales resultados obtenidos en el estudio.

\section{a) Caracterización de las familias}

Como dato que resulta importante para la caracterización sociodemográfica de las familias de los participantes, los padres también 
Apoyo familiar en escolares de alta capacidad intelectual de diferentes contextos... / Castellanos et al.

completaron un cuestionario de apoyo familiar (cuyos resultados no se reportan en este trabajo), aunque debe resaltarse que la mayoría se negó a aportar datos sobre su economía, condiciones materiales de vida e ingresos, lo cual se explica por la situación de inseguridad especial en el país. A partir de información que pudo recolectarse, y de lo reportado por los alumnos, se obtuvo la caracterización general del contexto familiar.

En la escuela rural, el 60\% de los padres que completaron el cuestionario expresó haber cursado solo el nivel de educación primaria o secundaria, mientras que el $20 \%$ restante ha alcanzado estudios técnicos, y otro $20 \%$ preparatoria. Mientras, en la escuela urbana pública, el $38.1 \%$ de los padres reportó haber concluido la educación primaria o la secundaria (14.3\% y $38.1 \%$ respectivamente), el $29.6 \%$ cursó la preparatoria o alguna carrera técnica, y el $33.4 \%$ de ellos tiene estudios universitarios, de estos últimos un $4.8 \%$ ha cursado algún posgrado.

Finalmente, solo el $6.5 \%$ de los padres de la escuela urbana privada que respondieron el cuestionario dijo haber terminado como mínimo la educación secundaria; el $40.9 \%$, la preparatoria, alguna carrera técnica o haber comenzado la universidad sin terminarla, mientras que un $53.3 \%$ de los mismos tiene como mínimo una carrera universitaria, algunos con estudios de posgrado (maestría o doctorado).

El 71.9\% de los alumnos participantes viven con ambos padres, y un $22.8 \%$ tiene uno o más hermanos que conviven en el hogar. En el $41.4 \%$ de los nińos que respondieron, la madre no trabaja fuera de la casa, mientras que solo el $9 \%$ declara que el padre no lo hace. La gran mayoría de los padres $(70.1 \%)$ permanece trabajando fuera del hogar el día entero o una parte importante de este, mientras que la proporción es más baja (42.2\%) en las madres de familia. Los niños reportan que en general, los padres dedican poco tiempo al acompańamiento o ayuda en tareas escolares: 30 minutos o menos en más de la mitad de los participantes $(53.5 \%$ para la asignatura de Espańol y $52.8 \%$ para Matemática). Solo un $4.2 \%$ y un $1.4 \%$ declaran que en la familia se dedica 2 horas diarias o más a apoyar en solución de las tareas de Español y de Matemática respectivamente. 


\section{b) Altas capacidades según resultados obtenidos en la prueba SAGES-2}

La Tabla 2 presenta las puntuaciones obtenidas a partir de la aplicación del SAGES-2 por subescalas. Como se observa, los promedios en las puntuaciones cocientes son más elevados en la subescala 3 de Razonamiento, y los más bajos se presentan en la subescala 2, de base lingüística-verbal (Lengua/Lit/Ciencias sociales).

\section{Tabla 2}

Estadisticas descriptivas para las subescalas del SAGES-2 por indicadores (cocientes y percentiles) en muestra $(n=79)$

\begin{tabular}{llcccc}
\hline Medidas (subescalas SAGES-2) & Mínimo & Máximo & $M$ & DE \\
\hline Cocientes & Matemáticas y & & & & \\
& Ciencias Naturales & 63 & 149 & 119.34 & 14.99 \\
& Lengua y Literatura, & 65 & 145 & 110.57 & 16.03 \\
& $\begin{array}{l}\text { Ciencias Sociales } \\
\text { Razonamiento }\end{array}$ & 73 & 140 & 119.11 & 11.90 \\
\cline { 2 - 6 } Percentiles & Matemáticas y & 1 & 99 & 83.15 & 22.89 \\
& $\begin{array}{l}\text { Ciencias Naturales } \\
\text { Lengua y Literatura, }\end{array}$ & 1 & 99 & 69.15 & 27.72 \\
& Ciencias Sociales & & & & \\
& Razonamiento & 4 & 99 & 85.33 & 17.92 \\
\hline
\end{tabular}

Las puntuaciones percentiles, que reflejan la posición relativa de los sujetos, son bajas, lo cual revela diferencias importantes entre la población a la cual ha sido aplicada la prueba, y la población en la cual fue estandarizada, aspecto que debe ser analizado en detalle en los marcos de la investigación posterior.

En relación con el sexo, solo se encontraron diferencias estadísticamente significativas entre hombre y mujeres $\left(t_{(77)}=2.46, p=.016\right)$ en la subescala 2 (Lengua, Literatura y Ciencias Sociales) a favor de las mujeres $(M=115.63, D E=13.9)$ sobre los hombres $(M=107.13$, $D E=16.6)$. 
La Tabla 3 muestra los resultados del SAGES-2 por contexto educativo. En las tres subescalas del SAGES, los mejores resultados se obtienen en la escuela urbana privada, y a su vez, se mantiene la tendencia a que los resultados de la pública urbana sean superiores a los de la escuela rural (indígena). Sin embargo, aunque las diferencias entre la escuela privada y las dos restantes son significativas en las tres subescalas, a favor de la primera, las diferencias entre la escuela urbana pública y la rural solamente son significativas en la subescala 2 (Lingüística) a favor de la primera, mientras que en la escala de Razonamiento, no resultaron significativas las diferencias entre estos dos tipos de escuelas.

\section{Tabla 3}

Puntuaciones Cocientes en el SAGES-2 por subescala y contextos/tipo de escuelas

\begin{tabular}{lcccc}
\hline $\begin{array}{l}\text { Contexto } \\
\text { socioeducativo }\end{array}$ & $\begin{array}{c}\text { Subescala 1. } \\
\text { Matemáticas y } \\
\text { Ciencias Naturales }\end{array}$ & $\begin{array}{c}\text { Subescala 2. } \\
\text { Lengua y } \\
\text { Literatura, } \\
\text { Ciencias Sociales }\end{array}$ & $\begin{array}{c}\text { Subescala 3. } \\
\text { Razonamiento }\end{array}$ \\
\hline Privado & $M$ & $104.83^{*}$ & $102.56^{*}$ & $106.56^{*}$ \\
$(\mathrm{n}=35)$ & $D E$ & 18.706 & 18.395 & 19.635 \\
Público urbano & $M$ & 90.70 & $\mathbf{8 8 . 2 2 ^ { * }}$ & 96.92 \\
$(\mathrm{n}=31)$ & $D E$ & 14.308 & 15.573 & 15.318 \\
Rural indígena & $M$ & 90.58 & $\mathbf{8 3 . 2 0 ^ { * }}$ & 93.01 \\
(n=13) & $D E$ & 13.925 & 13.152 & 16.580 \\
Total & $M$ & 93.58 & 88.81 & 97.06 \\
$(\mathrm{~N}=79)$ & $D E$ & 16.151 & 16.783 & 17.584
\end{tabular}

Nota: * las diferencias son significativas entre los grupos (contextos), $p<.05$

\section{c) Percepción de Apoyo Familiar en los alumnos}

La Tabla 4 presenta los porcentajes de respuestas de los alumnos de los tres contextos socioeducativos en cada una de las dimensiones, en Español y Literatura; se muestran las respuestas agrupadas en tres categorías según 
la frecuencia con que creen que sus padres desarrollan las actividades de apoyo: Baja (incluyen las respuestas en los valores Nunca o Casi nunca), Media (Algunas veces) y Alta (Casi siempre y Siempre). Puede notarse que en general un porcentaje elevado de alumnos perciben una frecuencia relativamente elevada de actividades de apoyo por parte de los padres, y que es bastante consistente esta percepción entre una y otra asignatura.

\section{Tabla 4}

Percepción la frecuencia de las actividades de apoyo familiar en las diferentes dimensiones y materias

\begin{tabular}{|c|c|c|c|c|c|}
\hline & & $\%$ & dentro de cad & la dimensi & ión $(\mathrm{N}=79)$ \\
\hline & & & $\begin{array}{c}\text { Baja } \\
\text { (Nunca o } \\
\text { Casi nunca) }\end{array}$ & $\begin{array}{c}\text { Media } \\
\text { (A veces) }\end{array}$ & $\begin{array}{c}\text { Alta } \\
\text { (Casi } \\
\text { siempre o } \\
\text { Siempre) }\end{array}$ \\
\hline & & $\begin{array}{l}\text { Asistencia- } \\
\text { Apoyo en Tareas }\end{array}$ & 38.9 & 6 & 54.2 \\
\hline Dimenciones & & $\begin{array}{l}\text { Evaluación y } \\
\text { Repaso }\end{array}$ & 29.1 & 7.3 & 66.3 \\
\hline $\begin{array}{l}\text { Apoyo } \\
\text { Familiar }\end{array}$ & Español & $\begin{array}{l}\text { Proporcionar } \\
\text { Tiempo y Espacio }\end{array}$ & 22.5 & 8.5 & 69 \\
\hline & & $\begin{array}{l}\text { Mantener } \\
\text { Comunicación } \\
\text { Regular con } \\
\text { Maestros }\end{array}$ & 34.3 & 8.6 & 57.1 \\
\hline & & $\begin{array}{l}\text { Asistencia- } \\
\text { Apoyo en Tareas }\end{array}$ & 41.2 & 4.4 & 54.4 \\
\hline & & $\begin{array}{l}\text { Evaluación y } \\
\text { Repaso }\end{array}$ & 22.4 & 12.1 & 65.5 \\
\hline & Matemática & $\begin{array}{l}\text { Proporcionar } \\
\text { Tiempo y Espacio }\end{array}$ & 26.2 & 7 & 66.2 \\
\hline & & $\begin{array}{l}\text { Mantener } \\
\text { Comunicación } \\
\text { Regular con } \\
\text { Maestros }\end{array}$ & 34.3 & 2.9 & 62.9 \\
\hline
\end{tabular}


Para examinar de manera más puntual las diferencias existentes entre los alumnos provenientes de los 3 contextos en las dimensiones del apoyo familiar se realizó un análisis de varianza simple con un factor, tipo de escuela o contexto, para cada una de las dimensiones por separado, en Español y Matemática. La Tabla 5 presenta las puntuaciones promedios en las ocho dimensiones de Apoyo familiar ( 4 para cada materia) por contexto. Se encontraron diferencias significativas entre los tres contextos educativos (privado, público urbano, público rural) aunque solo en una de las dimensiones del Apoyo Familiar, Mantener Comunicación Regular con Maestros (Español).

\section{Tabla 5}

Medidas de Apoyo Familiar por Contexto: Privada ( $n=34)$, Pública ( $n=27)$, E Rural Indigena $(n=11)$

\begin{tabular}{|c|c|c|c|c|c|}
\hline \multicolumn{2}{|c|}{ Dimensiones de Apoyo Familiar } & \multirow{2}{*}{$\begin{array}{c}M \\
2.13\end{array}$} & \multirow{2}{*}{$\begin{array}{c}D E \\
.81\end{array}$} & \multirow{2}{*}{$\begin{array}{c}\text { Mínimo } \\
.00\end{array}$} & \multirow{2}{*}{$\begin{array}{c}\text { Máximo } \\
3.83\end{array}$} \\
\hline & Urbano privado & & & & \\
\hline o apoyo en & Urbano público & 2.04 & 1.04 & .33 & 3.67 \\
\hline tareas escolares & Rural indígena & 1.88 & .58 & 1.17 & 2.83 \\
\hline & Total & 2.06 & .87 & .00 & 3.83 \\
\hline \multirow{4}{*}{$\begin{array}{l}\text { Asistencia } \\
\text { o apoyo en } \\
\text { tareas escolares } \\
\text { (Matemáticas) }\end{array}$} & Urbano privado & 2.27 & .75 & .67 & 3.67 \\
\hline & Urbano público & 2.17 & .97 & .33 & 3.67 \\
\hline & Rural indígena & 1.81 & .65 & .67 & 2.83 \\
\hline & Total & 2.17 & .83 & .33 & 3.67 \\
\hline \multirow{4}{*}{$\begin{array}{l}\text { Proporcionar } \\
\text { Tiempo y } \\
\text { Espacio para } \\
\text { el Estudio } \\
\text { (Español) }\end{array}$} & Urbano privado & 2.70 & .73 & 1.25 & 4.00 \\
\hline & Urbano público & 2.61 & 1.01 & .25 & 4.00 \\
\hline & Rural indígena & 2.37 & .75 & 1.50 & 4.00 \\
\hline & Total & 2.61 & .84 & .25 & 4.00 \\
\hline \multirow{4}{*}{$\begin{array}{l}\text { Proporcionar } \\
\text { Tiempo y } \\
\text { Espacio para } \\
\text { el Estudio } \\
\text { (Matemáticas) }\end{array}$} & Urbano privado & 2.73 & .79 & 1.25 & 4.00 \\
\hline & Urbano público & 2.51 & 1.06 & .00 & 4.00 \\
\hline & Rural indígena & 2.40 & .79 & 1.75 & 4.00 \\
\hline & Total & 2.60 & .89 & .00 & 4.00 \\
\hline
\end{tabular}




\begin{tabular}{llcccc}
\hline Dimensiones de Apoyo Familiar & $M$ & $D E$ & Mínimo & Máximo \\
\hline Mantener & Urbano privado & 2.38 & .71 & 1.00 & 3.80 \\
Comunicación & Urbano público & 2.32 & .81 & 1.00 & 3.80 \\
$\begin{array}{l}\text { Regular con } \\
\text { los Maestros }\end{array}$ & Rural indígena & 1.65 & .89 & .40 & 3.60 \\
y Directores & Total & 2.24 & .81 & .40 & 3.80 \\
(Espańol) & & & & & \\
\hline Mantener & Urbano privado & 2.34 & .72 & 1.00 & 3.75 \\
Comunicación & Urbano público & 2.31 & .85 & .80 & 3.80 \\
Regular con & Rural indígena & 1.80 & .85 & .40 & 3.60 \\
los Maestros & Total & 2.25 & .80 & .40 & 3.80 \\
y Directores & & & & \\
(Matemáticas ) & Urbano privado & 1.95 & .92 & .25 & 3.75 \\
\hline Evaluación & Urbano público & 2.19 & 1.27 & .00 & 4.00 \\
y Repaso & Rural indígena & 2.06 & 1.03 & .50 & 3.75 \\
(Espańol) & Total & 2.06 & 1.07 & .00 & 4.00 \\
\hline & Urbano privado & 2.21 & .97 & .00 & 3.75 \\
Evaluación & Urbano público & 2.12 & 1.16 & .00 & 4.00 \\
y Repaso & Rural indígena & 2.10 & .98 & 1.00 & 3.75 \\
(Matemáticas) & Total & 2.16 & 1.03 & .00 & 4.00 \\
\hline
\end{tabular}

*Nota: los valores oscilan entre $0-4$. A mayor valor, es mayor la frecuencia con que perciben el apoyo en las tareas señaladas.

El cálculo del ANOVA con un factor (Contexto) señaló la existencia de diferencias significativas solo en una de las dimensiones, Mantener Comunicación Regular con Maestros en Español ( $\mathrm{F}=3.74, p<.029)$, mientras que las pruebas post hoc de Comparaciones Múltiples de Bonferroni indicaron que las diferencias significativas se localizan entre el Contexto Privado y el Contexto Rural Indígena a favor del primero $(p=.029)$, y entre el Público Urbano y el Rural, a favor de este último $(p=.026)$. No hay diferencias significativas entre el contexto público urbano y el privado. En resumen, los alumnos de la escuela privada 
Apoyo familiar en escolares de alta capacidad intelectual de diferentes contextos... / Castellanos et al.

perciben una comunicación familia-escuela más frecuente que los de la escuela rural en lo relacionado con la materia de Español, y estos a su vez, perciben mayor frecuencia en esta comunicación que los de la escuela pública (ver Tabla 6).

\section{Tabla 6}

Resultados del ANOVA para las dimensiones del Apoyo Familiar como variables dependientes y el factor Contexto (Privado, Público Urbano y Rural)

\begin{tabular}{|c|c|c|c|c|c|c|}
\hline & & $\begin{array}{l}\text { Suma de } \\
\text { cuadrados }\end{array}$ & $g l$ & $\begin{array}{l}\text { Media } \\
\text { cuadrática }\end{array}$ & $F$ & $p$ \\
\hline \multirow{3}{*}{$\begin{array}{l}\text { Asistencia o } \\
\text { apoyo en tareas } \\
\text { escolares en } \\
\text { español }\end{array}$} & Intergrupos & .50 & 2 & .25 & \multirow[t]{3}{*}{.33} & \multirow[t]{3}{*}{.72} \\
\hline & Intragrupos & 53.97 & 69 & .78 & & \\
\hline & Total & 54.48 & 71 & & & \\
\hline \multirow{3}{*}{$\begin{array}{l}\text { Asistencia o } \\
\text { apoyo en tareas } \\
\text { escolares en } \\
\text { Matemáticas }\end{array}$} & Intergrupos & 1.77 & 2 & .89 & \multirow[t]{3}{*}{1.27} & \multirow[t]{3}{*}{.29} \\
\hline & Intragrupos & 47.96 & 69 & .69 & & \\
\hline & Total & 49.73 & 71 & & & \\
\hline \multirow{3}{*}{$\begin{array}{l}\text { Proporcionar } \\
\text { el Tiempo y } \\
\text { Espacio para } \\
\text { el Estudio en } \\
\text { Español } \\
\end{array}$} & Intergrupos & .86 & 2 & .43 & \multirow[t]{3}{*}{.59} & \multirow[t]{3}{*}{.56} \\
\hline & Intragrupos & 49.59 & 68 & .73 & & \\
\hline & Total & 50.46 & 70 & & & \\
\hline \multirow{3}{*}{$\begin{array}{l}\text { Proporcionar } \\
\text { el Tiempo y } \\
\text { Espacio para } \\
\text { el Estudio en } \\
\text { Matemáticas } \\
\end{array}$} & Intergrupos & 1.17 & 2 & .59 & \multirow[t]{3}{*}{.72} & \multirow[t]{3}{*}{.49} \\
\hline & Intragrupos & 55.52 & 68 & .87 & & \\
\hline & Total & 56.69 & 70 & & & \\
\hline \multirow{3}{*}{$\begin{array}{l}\text { Mantener } \\
\text { Comunicación } \\
\text { regular con } \\
\text { los Maestros y } \\
\text { Directores en } \\
\text { Español }\end{array}$} & Intergrupos & 4.57 & 2 & 2.28 & \multirow[t]{3}{*}{3.75} & \multirow[t]{3}{*}{$.03^{*}$} \\
\hline & Intragrupos & 40.82 & 67 & .61 & & \\
\hline & Total & 45.39 & 69 & & & \\
\hline
\end{tabular}




\begin{tabular}{lllllll}
\hline & & $\begin{array}{l}\text { Suma de } \\
\text { cuadrados }\end{array}$ & $g l$ & $\begin{array}{l}\text { Media } \\
\text { cuadrática }\end{array}$ & $F$ & $p$ \\
\hline Mantener & Intergrupos & 2.61 & 2 & 1.30 & 2.07 & .13 \\
$\begin{array}{l}\text { Comunicación } \\
\text { regular con }\end{array}$ & Intragrupos & 42.16 & 67 & .63 & & \\
$\begin{array}{l}\text { los Maestros y } \\
\text { Directores en }\end{array}$ & Total & 44.77 & 69 & & & \\
Matemáticas & & & & & & \\
\hline Evaluación & Intergrupos & .86 & 2 & .43 & .36 & .69 \\
y repaso en & Intragrupos & 81.11 & 69 & 1.18 & & \\
Español & Total & 81.97 & 71 & & & .08 \\
\hline Evaluación & Intergrupos & .17 & 2 & .085 & .93 \\
y repaso en & Intragrupos & 76.45 & 69 & 1.19 & & \\
Matemáticas & Total & 76.62 & 71 & & & \\
\hline
\end{tabular}

${ }^{*}$ Nota: $p<.05$

No se presentaron diferencias estadísticas entre hombres y mujeres en ninguna de las dimensiones del instrumento.

\section{d) Correlaciones entre las medidas de aptitud intelectualy percepción de apoyo familiar}

Para explorar en mayor detalle la existencia de asociaciones entre aptitud intelectual sobresaliente y apoyo, se calcularon las correlaciones bivariadas entre ellas, tomando por separado las materias de Español y Matemáticas.

Los resultados señalan la existencia de correlaciones significativas, pero negativas, entre la Subescala 2 (Lingüística) de la SAGES-2, y la percepción de apoyo en la dimensión de Evaluación y Repaso en Español $(r=-.45, p=.038)$. También, se observó la presencia de asociaciones positivas entre puntuaciones cocientes de la Subescala 3 (Razonamiento) y Proporcionar Tiempo y Espacio para el Estudio en Español $(r=.27, p=.021)$. En síntesis, la más alta capacidad en el área lingüística verbal se presenta asociada a la percepción de que los 
padres repasan y evalúan sus tareas de Español con menor frecuencia, y habilidades más elevadas en el área de Razonamiento, se asocia con la percepción de una mayor frecuencia de actividades relacionadas con proporcionar condiciones para el estudio.

Por otra parte, del cálculo de las correlaciones bivariadas de Spearman para los tres contextos socioeducativos de manera independiente, se obtuvo lo siguiente: en el contexto privado, no se evidenciaron correlaciones significativas entre las medidas de subescalas capacidad intelectual y las de apoyo familiar. Sin embargo, en la escuela pública se encontraron correlaciones moderadas pero positivas entre Razonamiento y Proporcionar Tiempo y Espacio para el estudio del Espańol $(r=.45, p=.02)$, mientras que en la rural indígena, se encontró una asociación moderadamente alta, negativa y significativa entre Razonamiento y Mantener comunicación con maestros y padres en Español $(r=-.65, p=.031)$.

\section{Discusión}

El propósito general de este trabajo fue comparar la percepción que los alumnos con capacidades sobresalientes provenientes de tres contextos educativos diferentes tienen acerca de la frecuencia con que sus padres desarrollan actividades de apoyo a su aprendizaje escolar en el hogar. Un aspecto adicional que emerge del propio estudio es la cuestión relativa a la manifestación de estas altas capacidades en dichos contextos a partir de su evaluación por la prueba SAGES-2.

En relación con los resultados obtenidos a partir de la aplicación de la prueba SAGES-2 en los participantes, puede decirse que, aunque no se examinaron las medidas de desempeño académico en los participantes (en este estudio se evaluó solamente la aptitud intelectual sobresaliente), los resultados que se reportan en este trabajo son en línea general consistentes con lo que muestran los estudios sobre logro académico en contextos educativos diferentes, que seńalan la ventaja de las escuelas privadas sobre las públicas, y las rurales indígenas (Backhoff, 2011). El fuerte contenido 
curricular de las subescalas 1 y 2, conformadas por tareas intelectuales que están saturadas de conocimiento previo en las áreas de Matemáticas / Ciencias Naturales, y Lengua y Literatura/ Humanidades, respectivamente, puede estar dando cuenta de este hecho.

Los análisis posteriores en los que se establezca la relación entre estas medidas y las medidas de logro o desempeño académico en los estudiantes, arrojarán mayor claridad sobre estos datos. La prueba SAGES-2 no resulta ser una prueba "libre" de influencia cultural, más bien, parece estar reproduciendo los resultados que se obtienen cuando se evalúa el capital cultural escolar en su influencia sobre el logro académico. Sus escalas, en particular aquellas desarrolladas para evaluar aptitudes académicas fundamentalmente relacionadas con el área lingüística y con la matemática, están saturadas de información y de contenidos culturales, que por otra parte, deberían ser revisados para comprobar su validez ecológica en el contexto educativo mexicano actual.

Las diferencias en la escala de Razonamiento (más cercana a lo que miden las pruebas tradicionales de capacidad general o inteligencia fluida) a favor de la escuela privada, y la superioridad de la pública urbana sobre la rural, también parecen estar apuntando a los típicos resultados que muestran que se favorecen estos contextos socioeconómicos. Ello puede estar vinculado con la existencia de mayores oportunidades de acceso a la cultura, así como a prácticas y estrategias de estimulación cultural y de apoyo familiar más frecuentes y eficientes, cuestión que constituye el centro de la investigación. También estaría relacionado con las mayores posibilidades económicas de las familias en los contextos privados, en el entorno de este tipo de alumnos. Sin embargo, muchos de los estudios en el área muestran que no es necesariamente el nivel socioeconómico familiar determinante en el desarrollo académico de los estudiantes. Otros aspectos culturales pueden estar influyendo, como el entrenamiento en el tipo de pruebas, que es una práctica común en los colegios privados y no en los otros tipos de centros educativos.

En síntesis, lo que parece estar asociado al nivel socioeconómico familiar en este estudio es la probabilidad que tienen los hijos de poder ser 
Apoyo familiar en escolares de alta capacidad intelectual de diferentes contextos... / Castellanos et al.

detectado como estudiantes con altas capacidades en una o más áreas de desempeño, como muestran los resultados que favorecen prácticamente en todos los casos, al contexto de la escuela privada. De ser identificados con este tipo de instrumentos los alumnos con aptitudes sobresalientes, quedan en franca desventaja — desventaja socioeconómica- los alumnos del contexto rural indígena. Este hecho es congruente con lo planteado por autores como Arencibia (2010) acerca de la importancia de un enfoque de sensibilidad cultural como requisitos de la toma de decisiones sobre las estrategias de identificación del potencial de los alumnos en los diferentes contextos y tipos de escuelas.

Por otra parte, de acuerdo con los datos obtenidos en el presente estudio, el nivel socioeconómico (evaluado de manera indirecta por el contexto socioeducativo) no parece estar asociado por sí mismo de manera significativa con las percepciones que tienen los alumnos con alta capacidad sobre el apoyo familiar que reciben de los padres de familia. Solamente se obtuvieron diferencias significativas en la dimensión de Mantener Comunicación Regular con los maestros y directivos con una mayor frecuencia en el contexto privado, que estaría seńalando una mayor participación e involucramiento de la familia en estos entornos, y en el indígena sobre el contexto público. El examen detallado de la expresión de involucramiento en las escuelas rurales debe ser abordado en mayor detalle tomando en consideración la vida y la cultura escolar en estas comunidades.

En sentido general, los resultados no son claramente congruentes con lo encontrado por algunos autores (Alonso \& Benito, 1997; Castellanos et al., 2004; Freeman, 2006) acerca de la valoración positiva que los alumnos sobresalientes expresan sobre el apoyo familiar. En este estudio, aunque muchos de los alumnos percibieron una frecuencia relativamente alta de apoyo familiar, un porcentaje también elevado ve que la frecuencia de estas actividades es pobre o nula, lo que estaría más en línea con lo reportado por Hernández y Borges (2010), quienes encontraron incluso valoraciones negativas o muy pobres en los alumnos al opinar sobre el apoyo familiar en sus hogares: los adolescentes veían a sus padres preocupados por sus actividades y logros académicos, pero 
también pensaban que estos propician independencia en la realización de sus tareas; tampoco perciben alto grado de comunicación con la escuela.

Por otra parte, sería necesario examinar con mayor profundidad la relación de este hecho con el nivel cultural más elevado de los padres (superior en el contexto de la escuela privada como se evidenció en la caracterización de las familias), que podría vincularse a su vez, con las mayores expectativas y creencias de estos sobre su capacidad o competencia para ayudar a sus hijos sobresalientes, y consecuentemente, con su motivación para poner en práctica diferentes actividades de apoyo. También es necesario tomar en cuenta que los estilos de interacción que se construyen en la dinámica familiar (dentro de los cuales se encuentran estas actividades compartidas de apoyo al aprendizaje), no surgen de forma unilateral por la pura voluntad o intención de los padres. Como sostiene Palacios (1999), el niño no es un ente pasivo, sino que de manera activa se involucra en estas dinámicas, definiendo de manera singular la dirección de las mismas y cómo se expresan en la vida cotidiana de la familia. La investigación previa muestra que las prácticas parentales en este sentido dependen en mucho de las características de los hijos, y en este caso, la mayor autonomía o independencia de los alumnos con alta capacidad intelectual en el campo académico, puede estar condicionando un menor involucramiento de la familia en las actividades de apoyo, o al menos, un menor involucramiento en las actividades que han quedado definidas en el marco de este estudio.

Quedaría por investigar aquellas estrategias concretas en las que sí participarían las familias para apoyar el desarrollo académico de sus hijos con alta capacidad intelectual. Y finalmente, queda también por indagar si las conductas o actividades de soporte al aprendizaje escolar en el hogar, o la baja frecuencia de ellas en algunos casos, son percibidas por los niños como estrategias para fomentar su independencia y autonomía como plantean Borges, Hernández y Rodríguez (2010), o como una falta de involucramiento o apoyo de sus familiar a su desarrollo y desempeño académico, cuestión que en esta investigación no ha quedado abordada.

Por último, se evidencia de este estudio la necesidad de indagar con mayor profundidad la naturaleza psicosocial y cultural de la influencia 
de los diferentes contextos educativos (a saber, socioeconómicos) en la estimulación del potencial de los niños, y en el consecuente desarrollo de capacidades y talentos (ver, por ejemplo, la influencia de variables contextuales como el apoyo familiar). El desarrollo de las altas capacidades se debe explicar desde modelos socioculturales multidimensionales, que rindan cuenta de su complejidad. Como plantean Plucker y Barab (2005), se trata de desarrollar esfuerzos desde la investigación para sustituir la visión de la capacidad intelectual superior como una posesión individual en lugar de un modo de funcionamiento que permite a la persona resolver problemas, producir y crear, en la interacción con un contexto y una situación concretos.

La problemática abordada en el trabajo es de relevancia actual, por cuanto indaga en la influencia del contexto socioeconómico en el apoyo familiar, y en la percepción de estimulación de las altas capacidades en estudiantes del nivel secundario. Al ser parte de una investigación más amplia, esta primera etapa confirma la importancia de profundizar en la caracterización de los contextos socioeducativos expresados aquí en el tipo de escuelas, de acuerdo con su localización y el nivel socioeconómico de la población escolar, y del entorno social en que se encuentra. Tomando en consideración que una de las limitantes del trabajo fue que solamente se reportan resultados de los alumnos identificados como sobresalientes, resulta relevante reportar en los futuros trabajos las comparaciones con estudiantes con diferentes niveles de capacidades y competencias.

Adicionalmente, es de gran interés continuar estudiando las percepciones que tienen los alumnos sobre los roles que están desempeñando sus familias en el apoyo a sus tareas y actividades académicas. Las relaciones entre apoyo familiar por una parte, y desarrollo de competencias y altas capacidades por otra, no son lineales; el trabajo muestra que es necesario trascender la visión superficial de las mismas, para incluir otras variables como la autonomía y la independencia desarrollada por los adolescentes, y la construcción de significados muy singulares que estarían reflejándose en sus valoraciones sobre el involucramiento y la ayuda que la familia les brinda. 


\section{Referencias}

Alacalay, L., Milicic, N. \& Torretti, A. (2005). Alianza Efectiva FamiliaEscuela: Un Programa Audiovisual Para Padres. Psykhe, 14(2), 149-161.

Alonso, J. \& Benito, Y. (1996). Superdotados: Adaptación escolar y social en Secundaria. Madrid: Narcea Ediciones.

Arencibia, V. (2010). Identificación y selección de alumnos talento en contextos de pobreza. En M. D. Valadez \& S. Valencia (Eds.), Desarrollo y educación del talento en adolescentes (pp. 224- 247). Universidad de Guadalajara: Editorial Universitaria.

Backhoff, E. (2011). La inequidad educativa en México. Profesorado. Revista del currículum y formación del profesorado, 15(3), 88-102. Backhoff, E., Sánchez, A., Peón, M. \& Andrade, E. (2010). Comprensión lectora y habilidades matemáticas de estudiantes de educación básica en México: 2000-2005. Revista Electrónica de Investigación Educativa, 12(1). Recuperado de http://redie.uabc. $\mathrm{mx} /$ vol12no1/contenido-backhoffsanchez.html

Bazán, A., Sánchez, B. \& Castañeda, S. (2007). Structural relationship among parents' support, parent's educational level, characteristics of the teacher and child's achievement in reading and writing. Revista Mexicana de Investigación Educativa, 3, 701-729.

Borges, A., Hernández, C. \& Rodríguez, E. (2006). Comportamientos parentales en familias con superdotados. Faísca, 11(13), 48-58.

Castellanos, D. (2003). Identificación de potencialidades intelectuales: la perspectiva del profesor. En D. Castellanos (Comp.), Talento: Estrategias para su Desarrollo (2da Edición) (pp. 33-45). La Habana: Editorial Pueblo y Educación.

Castellanos, D., Vera, C., Vera, N., López, C., Caballero... \& Rodríguez, R. (2004). Estudio de los factores asociados a la actualización del desempeño talentoso en estudiantes de la educación primaria, media y superior. Informe Técnico de Resultados. La Habana: Instituto Superior Pedagógico E. J. Varona. Plan de Ciencia e Innovación Tecnológica 2003-2005. MINED. 
Apoyo familiar en escolares de alta capacidad intelectual de diferentes contextos... / Castellanos et al.

Cervini, R. (2003). Relación entre composición escolar, proceso escolar y el logro en matemática del nivel secundario de Argentina. Revista Electrónica de Investigación Educativa, 5(1). Recuperado de http://redie.ens.uabc.mx/vol5no1/contenido-cervini2.html.

Colangelo, N. \& Assouline, S. (2000). Counseling gifted students. En K. A. Heller, F. J. Monks, Robert J. Sternberg \& F. Rena Subotnik (Eds.), International Handbook of Giftedness and Talent (2nd ed., pp.595-620). Amsterdam: Elsevier.

De la Torre, FG. \& Pérez, L. (2006). La familia y el desarrollo del potencial creativo de los niños con altas capacidades. En L. Pérez (Coord.), Alumnos con capacidad superior: Experiencias de intervención educativa (pp. 353-392). Madrid: Editorial Síntesis.

Domínguez, L. (2008). Apoyo familiar y desempeño en lengua escrita en escolares de quinto grado de primaria (Tesis de Licenciatura). Universidad Autónoma del Estado de Morelos, México.

Domínguez, L. (2011). Actividades de apoyo familiar y rendimiento académico en estudiantes de quinto grado de primaria (Tesis de maestría). Universidad Autónoma de Morelos, México.

Freeman, J. (2006). Un Estudio de Tres Décadas sobre Niños Superdotados y Talentosos. International Symposium, Gran Canaria, 2-4 noviembre, 2006.

Freeman, J. (2010). Families, the essential context for gifts and talents. En K. A. Heller, F. J. Monks, R. Sternberg \& R. Subotnik (Eds.), International Handbook of Research and Development of Giftedness and Talent (pp. 573-585). Oxford: Pergamon Press.

Gagné, F. (2010). Construyendo talentos a partir de la dotación: Breve revisión del MDDT 2.0. En M. D. Valadez \& S. Valencia (Comps.), Desarrollo y educación del talento en la adolescencia (pp. 64-78). Universidad de Guadalajara: Editorial Universitaria.

Gómez, M. A. \& Valadez, M. D. (2010). Relaciones de la familia y del hijo/a con superdotación intelectual. Faisca, 15(17), 67-85.

Grolnick, W. S. \& Slowiaczek, M. L. (1994). Parents' involvement in children's schooling: A multidimensional conceptualization and motivational model, Child Development, 64, 237-252. 
Hernández, C. \& Borges, A. (2010). Entorno escolar del alumnado de altas capacidades frente a sus compañeros de distinto nivel de inteligencia. Faisca, 12(17), 36-49.

Huerta, E. (2009). La relación escuela-padres en las secundarias mexicanas. Colección Cuadernos de Investigación (Cuaderno 34). Instituto Nacional para la Evaluación de la Educación (INEE), México.

Jiménez, C., Murga, M. G., Gil, J. A., Téllez, J. \& Trillo, M. P. (2010). Hacia un modelo sociocultural explicativo del alto rendimiento y la alta capacidad: Ámbito familiar. Educación, 21(13), 125-153. Madrid: Facultad de Educación. UNED.

Johnsen, S. \& Corn, A. (2003). SAGES-2:K3 Evaluación Inicial para Estudiantes con Aptitudes Sobresalientes (Educación primaria y secundaria). México: El Manual Moderno.

López-Aymes, G., Acuña, S. \& Durán, G. (2013). Families of Gifted Children and Counseling Program: A Descriptive Study in Morelos, Mexico. Journal of Curriculum and Teaching, 3(1), 54-62.

López-Aymes, G., Acuña, S. R. \& Mercado, A. (2013). Niños y niñas con altas capacidades intelectuales y sus familias. En A. Bazán \& C. Butto (Coords.), Psicología y contextos educativos. México: Horizontes Educativos. Universidad Pedagógica Nacional.

Martínez, F. (2004). La educación, la investigación educativa y la psicología. En S. Castañeda (Ed.), Educación, Aprendizaje y Cognición. Teoría en la práctica. México: Manual Moderno.

Martínez, M. \& Guirardo, A. (2012). Colaboración familia-comunidad educativa. En M. Martínez \& A. Guirardo (Coords.), Altas capacidades intelectuales. Pautas de actuación, orientación, intervención y evaluación en el periodo escolar (pp. 278-316). Barcelona: Editorial Grao.

Organización para la Cooperación y el Desarrollo Económicos (OCDE) (2006). Panorama de la educación 2006. Indicadores del $O C D E$. México: Santillana. 
Apoyo familiar en escolares de alta capacidad intelectual de diferentes contextos... / Castellanos et al.

Ordaz, G. \& Acle, GG. (2010). Resiliencia y aptitudes sobresalientes en niños de zonas urbano-marginadas. Revista Ideacción, 31, 228-299.

Orta, Y. \& Sánchez, P. (2003). Habilidades verbales de niños con habilidades sobresalientes: un estudio exploratorio preliminar en Yucatán (Artículo no publicado). Universidad Autónoma de Ciudad Juárez y Universidad Autónoma de Yucatán.

Palacios, J. (1999). La familia y su papel en el desarrollo afectivo y social. En E. López, I. Etxebarria, M. J. Fuentes \& J. Ortiz (Coords.), Desarrollo afectivo y social. Madrid: Ediciones Pirámide.

Pérez, L. (2004). Superdotación y familia. Faisca, 11, 17-36.

Plucker, A. \& Barab, S. (2005). The importance of contexts in theories of giftedness. Learning to embrace the messy joys of subjectivity. En R. J. Sternberg, \& J. E. Davison (Eds.), Conceptions of Giftedness (2nd. Ed.) (pp. 201-216). New York: Cambridge University Press.

Purkey, S. \& Smith, M. (1983). Effective schools: A review. Elementary School Journal, 83, 427-452.

Reimers, F. \& Cárdenas, S. (2007). Who benefits from school-based management in Mexico? Prospects, 37(1), 37-56.

Renzulli, J. S., \& Reis, S. M. (2000). The schoolwide enrichment model. En K. A. Heller, F. J. Monks, Robert J. Sternberg \& Rena F. Subotnik (Eds.), International Handbook of Giftedness and Talent (2nd ed., pp. 367-382). Amsterdam: Elsevier.

Rivas, B. S (2007) La participación de las familias en la escuela. Revista Española de Pedagogía, 238, 59-574.

Schmelkes, S. (2003). Escuelas, maestros y resultados de aprendizaje en América Latina. En: VI Congreso Nacional de Investigación Educativa. Conferencias Magistrales. México: COMIE, pp. 128-158.

Soriano, E. I. (2013). Concepciones de docentes y padres acerca de las aptitudes sobresalientes: Un estudio sociocultural en Morelos (Tesis de Licenciatura). Universidad Autónoma del Estado de Morelos, México. 
Soriano, E. I. \& Castellanos, D. (2014). Concepciones acerca de las aptitudes sobresalientes, su educación y desarrollo en padres de alumnos con aptitudes sobresalientes de dos escuelas secundarias del Estado de Morelos En K. P. Martínez \& H. Torres (Coords.), Investigación Educativa y Politicas Públicas: Formación Integral (Tomo II) (pp. 25-41). México: Universidad Autónoma de Nayarit.

Sternberg, R. (2001). Giftedness as developing expertise: a theory of the interface between high abilities and achieved excellence. High Ability Studies, 12(2), 159-179.

Valdés, A. A. \& Vera, J. A. \& Yañez, A. I. (2012). El papel de la familia en la atención a los estudiantes con aptitudes intelectuales sobresalientes. En A. A. Valdés \& J. A. Vera (Coords.), Estudiantes intelectualmente sobresalientes. México: Pearson Educación.

Ziegler, A. \& Heller, K. A. (2000). Conceptions of Giftedness from a Meta-Theoretical Perspective En K. A. Heller, F. J. Monks, R. J. Sternberg \& R. F. Subotnik (Eds.), International Handboook of Giftedness and Talent (2dn. Ed.) (pp. 3-22). Oxford: Pergamon.

Recibido: 07 de julio, 2014 Aceptado: 21 de noviembre, 2014 